\title{
Influence of blanking distance of materials on increase in blanking vibration using screw drive servo press
}

\author{
Tomohiro MURAKAMI*, Ming YANG**, Hiroshi KISHI*, \\ Sigeki MORI* and Masahiro OHKAWA* \\ *Polytechnic University \\ 2-32-1, Ogawa-Nishi-machi, Kodaira-shi, Tokyo 187-0035, Japan \\ E-mail: murakami@uitec.ac.jp \\ ** Graduate School of System Design, Tokyo Metropolitan University, \\ 6-6, Asahigaoka, Hino-shi, Tokyo, 191-0065, Japan
}

\section{Received 14 October 2015}

\begin{abstract}
In this study, we propose a suitable blanking velocity for the blanking of different materials using a screw drive servo press. Under this blanking velocity, press blanking can be carried out without causing an increase in blanking vibration. Blanking noise and vibration are causes of pollution. Moreover, blanking vibration reduces the precision of press work products and the lifetimes of the press machine and press die. Recently, a servo press has been developed for advanced press work. A screw drive servo press does not have a mechanical bottom dead center but location of its slide is constantly measured, and highly precise location and speed control are achieved by a feedback control system. However, blanking vibration is increased at high blanking velocities using this machine. Our previous study clarified that the blanking velocity and blanking time affect of blanking vibration. However, the influence of the material is not clear. Thus, we studied the relationship between the material and the vibration in blanking with a screw drive servo press both experimentally, and by simulation. At the experiment, we compared the blanking vibration of four materials with different tensile strengths. As a result, we found that the blanking time is different for each material, because the blanking distance depends on each material. And finally, we propose an equation as a general formula which calculates the threshold of blanking velocity $v_{t}$ to prevent an increase in blanking vibration.
\end{abstract}

Key words : Screw drive servo press, Tensile strength, Breaking load, Blanking distance, Blanking time, Break through distance, Feedback control, Disturbance settling time

\section{Introduction}

In recent years, servo presses have been developed for use by stamping companies. A screw drive servo press is one type of servo press, in which a slide is driven by transmitting the output of a servo motor to a feed screw. Therefore, a screw drive servo press does not have a specific bottom dead center, although the rated output can be applied at the optional location. In this machine, the good precision and parallelism of a slide is achieved by closed-loop feedback control of the slide location (Osakada et al., 2011). The mechanism of a screw drive servo press is shown in Fig 1. (Machida, et al., 2005) reported that a precise and complicated gear was sheared by progressive die using this kind of servo press.

On the other hand, some factories have reported that the blanking noise of a screw drive servo press is larger than that of a crank press. (Otsu, et al., 2003) reported that a blanking in low velocity has an effect on reduction of blanking noise. (Murakawa, et al., 2001) and (Ghiotti, et al., 2010) reported a technique of the reduction in blanking vibration by a damper. However, it was not clarified why a blanking noise was large using a screw drive servo press. To investigate this issue, (Murakami, et al., 2015) analyzed the vibration in blanking and confirmed increased vibration in the blanking of a screw 
drive servo press, due to the deviation of the slide position as follows. First, the punching load causes the deviation of the slide position. Then, with the deviation of the slide position, a control response force is applied to the slide, thus increasing the vibration. When the slide position does not deviate, this behavior does not occur, and thus Murakami clarified that the increase in vibration in blanking is affected by the response lag of the control system. Therefore, Murakami's paper suggests that the vibration is closely related to the blanking time. The blanking time is defined as the time from the contact of the punch with the material to the breakage of the material. Generally, the blanking load and rupture position depend on the strength and ductility of the material. Therefore, there are material differences that may affect the vibration of a screw drive servo press. However, this has not yet been studied.

In this study, we conducted a blanking experiment using materials with different strengths with a screw drive servo press. On the basis of the experimental results, we analyzed the relationship between the fracture condition and blanking vibration. We also clarified the influence of the material on the blanking vibration.

\section{Experimental procedure}

In the experiment, we used a screw drive servo press (KOMATSU HCP3000) with a rated pressure capacity of 800kN. Fig. 1 shows a schematic diagram of the machine. The structure of this machine is a straightside type as shown in Fig. 1(a). As shown in Fig. 1(b), this machine drives a slide by transmitting the output of a servo motor to a feed screw. The slide position is detected by a linear gauge mounted on each side of the slide, and a controller ensures the parallelism of the slides by adjusting the servo output. Because each slide is driven by a screw, its position and velocity can be changed arbitrary.

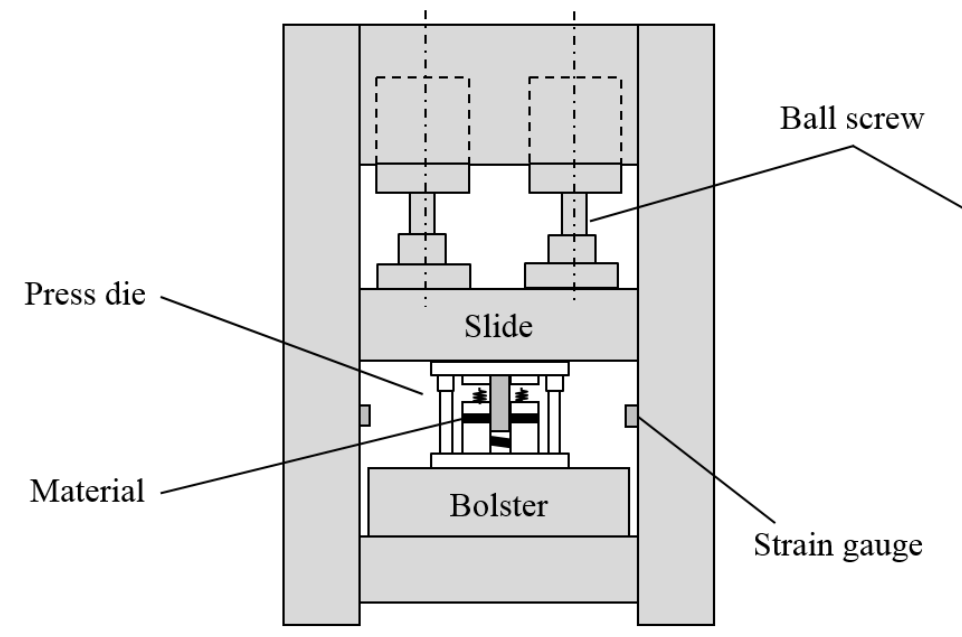

(a) Front view

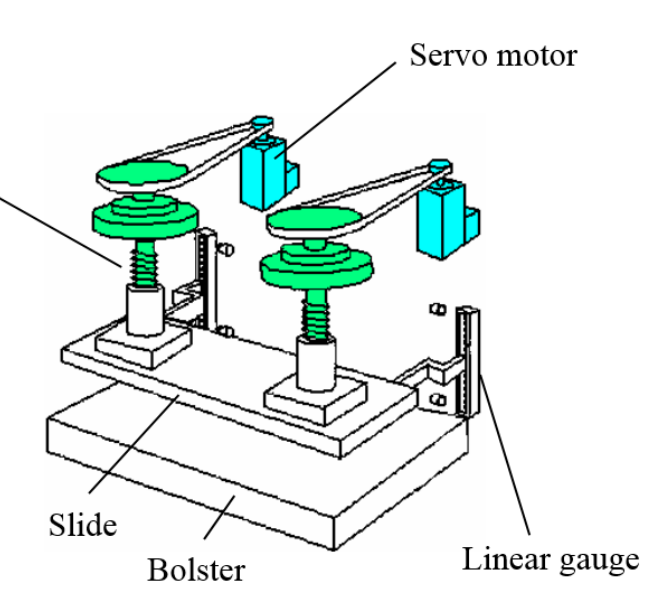

(b) Mechanism (Suzuki, 2001)

Fig. 1 Schematic diagram of screw drive servo press.

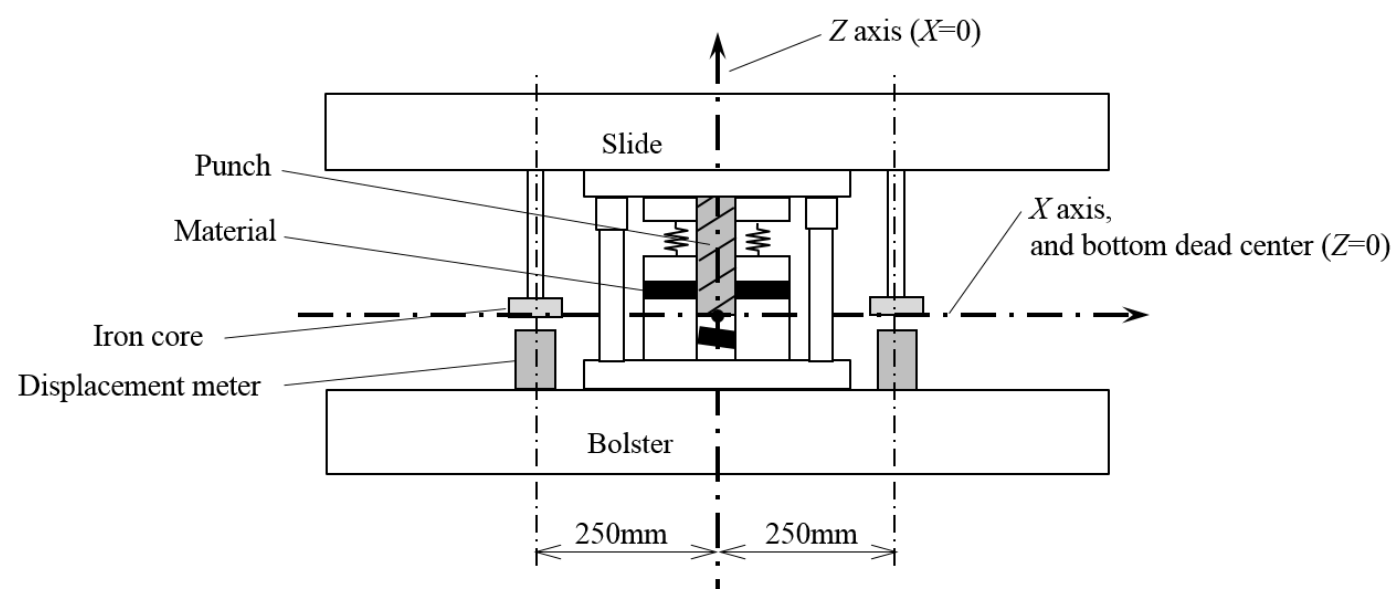

Fig. 2 Coordinate system and positions of press die and displacement meter. 
The blanking load is measured by a strain gauge mounted on the side wall of the press frame, as shown in Fig. 1(a). The output of the strain gauge is recorded using a digital oscillograph installed after an amplifier. The blanking load is the load between the slide and the bolster. The strain gauge as above is calibrated by a standard load generator, consisting of a load cell and a hydraulic jack.

We used a single blanking die in the experiment. The die was placed between the slide and bolster, as shown in Fig. 1(a). The size of the die hole was $40 \mathrm{~mm} \times 40 \mathrm{~mm}$, the corner radius was $1 \mathrm{~mm}$, and the die clearance was $5 \%$ of the thickness of $1 \mathrm{~mm}$. Fig. 2 shows the coordinate system superimposed on the press machine in this study. The center of the punched edge at the bottom dead center position was the origin of the coordinate system and the $X$ and $Z$ axes were set as shown in Fig. 2.

The blanking noise was measured with a sound level meter set as shown in Fig. 3. The sound pressure output (V) was recorded using a digital oscillograph. The position of the punch edge was measured by a displacement meter (sampling frequency: 20-50 kHz) whose location is shown in Fig. 2 and Fig. 3. The output of the displacement meter was also recorded in using the digital oscillograph.

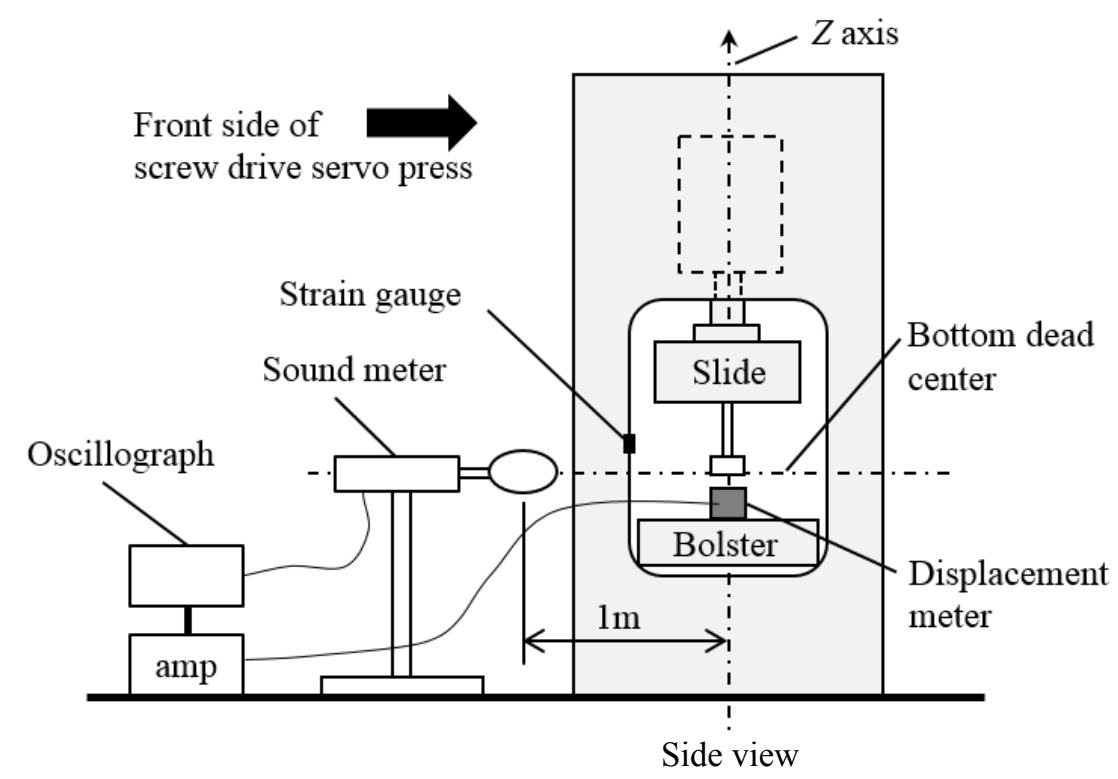

Fig. 3 Setting positions of measurement devices.

As specimens, we used four materials with different tensile strengths. The size of each specimen was $80 \mathrm{~mm} \times 80 \mathrm{~mm}$ and the materials were pure copper $\mathrm{C} 1100$ (Hereafter "Cu"), mild steel SPC-C (Hereafter "SPC"), 18Cr-8Ni stainless steel (Hereafter "SUS"), and high-strength steel SPFC980Y (Hereafter "SPFC"). The thicknesses of Cu, SPC, and SUS were 1 $\mathrm{mm}$ sand the thickness of SPFC was $1.2 \mathrm{~mm}$. The tensile strength of each material is shown in Table 1. As a specimen for a tensile test, we prepared a specimen of No. 13B (JIS Z2241:2011) in accordance with the tensile test method for metallic materials.

The blanking velocity was set at $V=15 \mathrm{~mm} / \mathrm{s}$ and $0.5 \mathrm{~mm} / \mathrm{s}$, where the former was used to confirm the phenomenon of increasing vibration, whereas no increase in the vibration was assumed for the latter. We defined the blanking velocity as the punch velocity immediately before breaking.

Table 1 Mechanical Properties of materials.

\begin{tabular}{|c|c|c|c|c|}
\hline material & $\mathrm{Cu}$ & SPC & SUS304 & SPFC \\
\hline $\mathrm{R}_{\mathrm{m}} \quad\left[\mathrm{N} / \mathrm{mm}^{2}\right]$ & 236 & 327 & 697 & 988 \\
\hline $\mathrm{A}_{50 \mathrm{~mm}} \quad[\%]$ & 50 & 45 & 57 & 15 \\
\hline
\end{tabular}

\section{Results and discussion}

\subsection{Blanking vibration and Breaking load}

Fig. 4(a) and Fig. 4(b) show the position of the punch edge and the sound pressure in the blanking of SPC, where the 
position of the punch edge is shown as the distance from the $Z$-axis origin, which is the bottom dead center of the press slide. The dot-dashed line shows the position of the punch edge when the material is not placed in the press die. Hereafter, we call this dot-dashed line the planned trajectory. The solid line shows the position of the punch edge when the material is punched, hereafter called the blanking trajectory. Fig. 4(c) shows the relationship between the planned trajectory and the blanking trajectory. Position p1 in Fig. 4(a) shows the position at the start of blanking. Fluctuations in the sound pressure were observed when the punch come in contact with the material. Position p2 in Fig. 4(a) shows the position of breakage. The blanking trajectory rapidly descended after reaching $\mathrm{p} 2$. This occurred simultaneously with a rapid increase in sound pressure, which is the source of the blanking noise. The blanking trajectory reached the bottom position p3 after descending. After that, the blanking trajectory reached the position on the planned trajectory while the vibration was damped. In this study, we defined the distance in the $Z$-axis direction from the position at the start of blanking $\mathrm{p} 1$ to the position of breakage $\mathrm{p} 2$ as the blanking distance (Hereafter " $l_{b}$ "), we defined the travel time for the $l_{b}$ as the blanking time (Hereafter " $t_{b}$ "), and we defined the distance in the $Z$-axis direction from position $\mathrm{p} 2$ to the bottom position $\mathrm{p} 3$ as the break through distance. But, a distance of the planned descent was excluded from the break through distance. Because, the planned descent is an amount of slide descent during in break through, and is unrelated to blanking vibration and is along the planned trajectory as shown in Fig. 4(a).

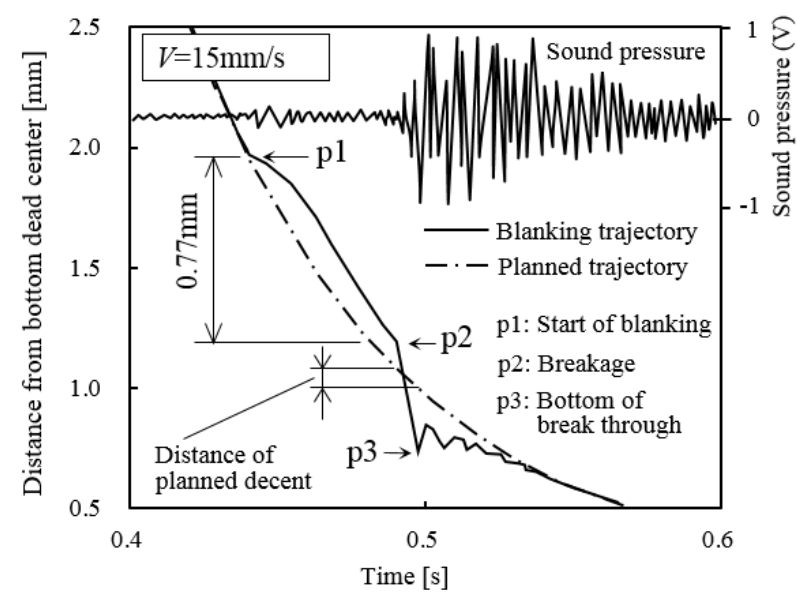

(a) $V=15 \mathrm{~mm} / \mathrm{s}(\mathrm{SPC})$

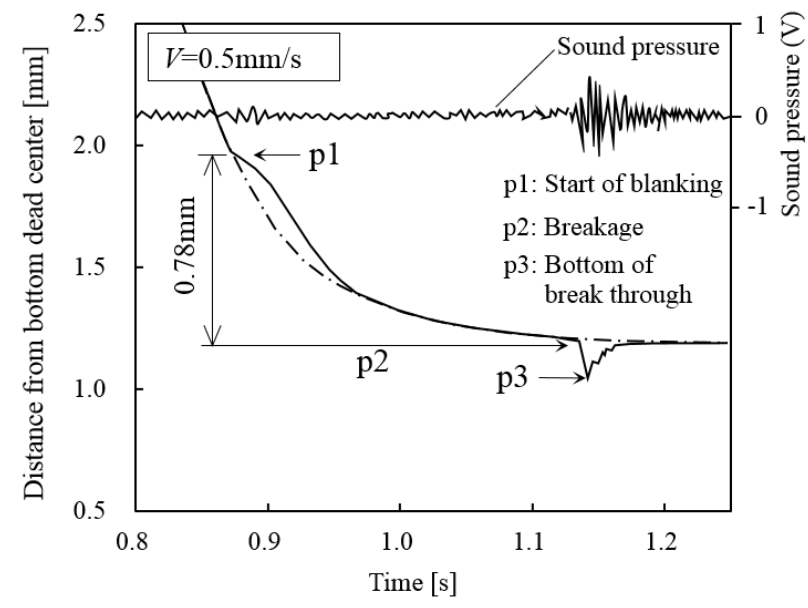

(b) $V=0.5 \mathrm{~mm} / \mathrm{s}$ (SPC)

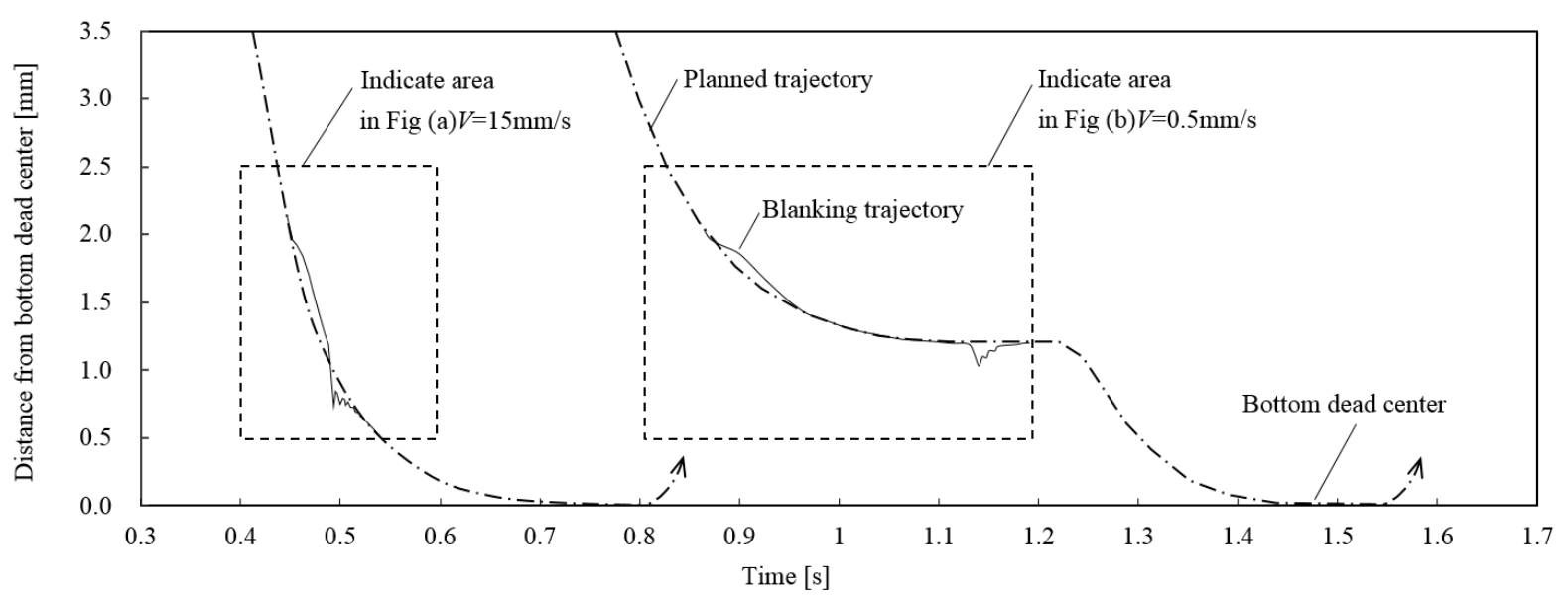

(c) Relationship between planned trajectory and blanking trajectory

Fig. 4 Punch trajectory and sound pressure in the blanking of SPC. Thickness of the specimen is $\mathrm{t}=1 \mathrm{~mm}$, the size of blanking products is $40 \mathrm{~mm} \times 40 \mathrm{~mm}$, and blanking by a screw drive servo press.

Fig. 4(b) shows the data obtained in the blanking of SPC with $V=0.5 \mathrm{~mm} / \mathrm{s}$. Similarly to in Fig. 4(a), we can clearly identify position $\mathrm{p} 1$ to $\mathrm{p} 3$. However, there are different points, in the cases of blanking with $V=0.5 \mathrm{~mm} / \mathrm{s} \mathrm{and} 15 \mathrm{~mm} / \mathrm{s}$. In the case of blanking with $V=0.5 \mathrm{~mm} / \mathrm{s}$, the blanking trajectory returned to the planned trajectory before reaching position 
p2. On the other hand, when $V$ was $15 \mathrm{~mm} / \mathrm{s}$, the blanking trajectory did not return to the planned trajectory before reaching position $\mathrm{p} 2$.

Fig. 5 shows the punch trajectory in the blanking of SUS. The blanking condition is same as Fig.4. Similarly to in Fig. 4, we can clearly identify position p1 to p3 from Fig. 5.

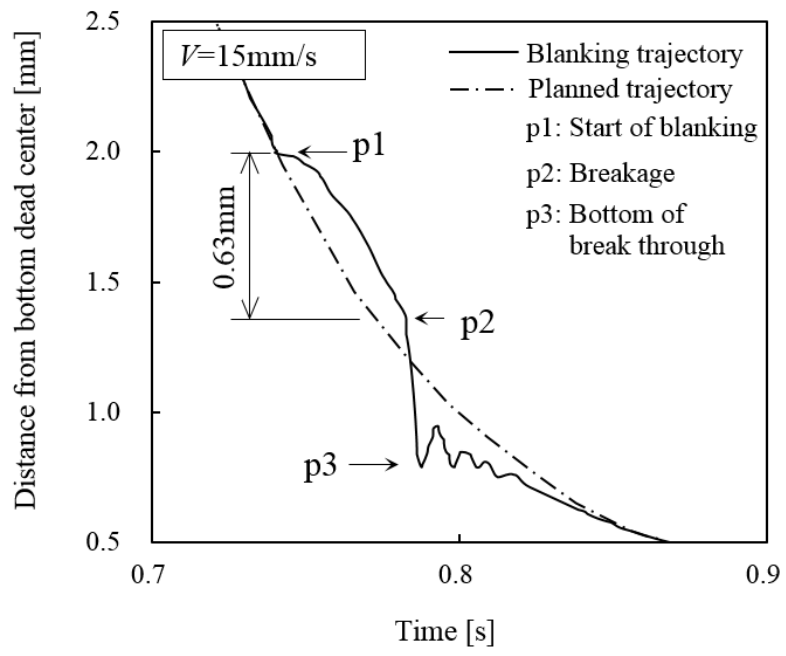

(a) $V=15 \mathrm{~mm} / \mathrm{s}$ (SUS)

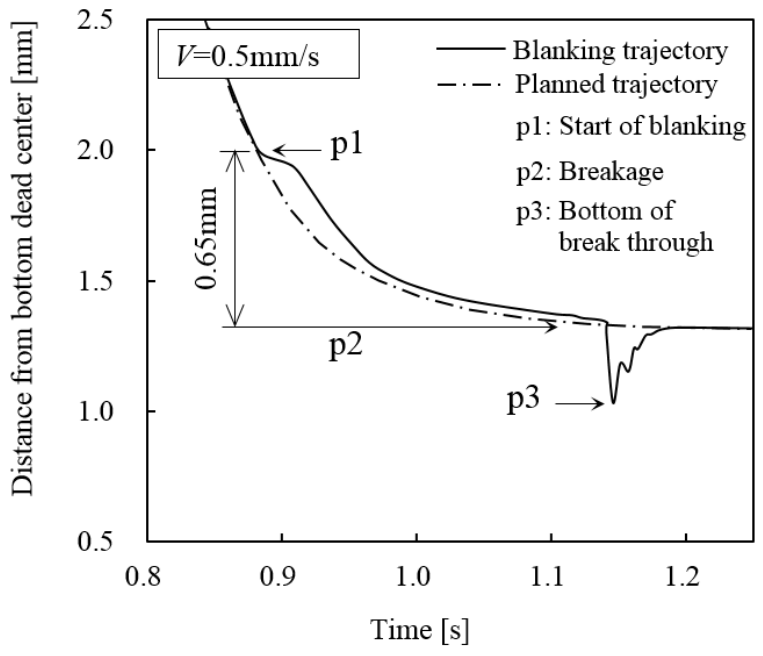

(b) $V=0.5 \mathrm{~mm} / \mathrm{s}$ (SUS)

Fig. 5 Punch trajectory in the blanking of SUS. The blanking condition is same as Fig.4.
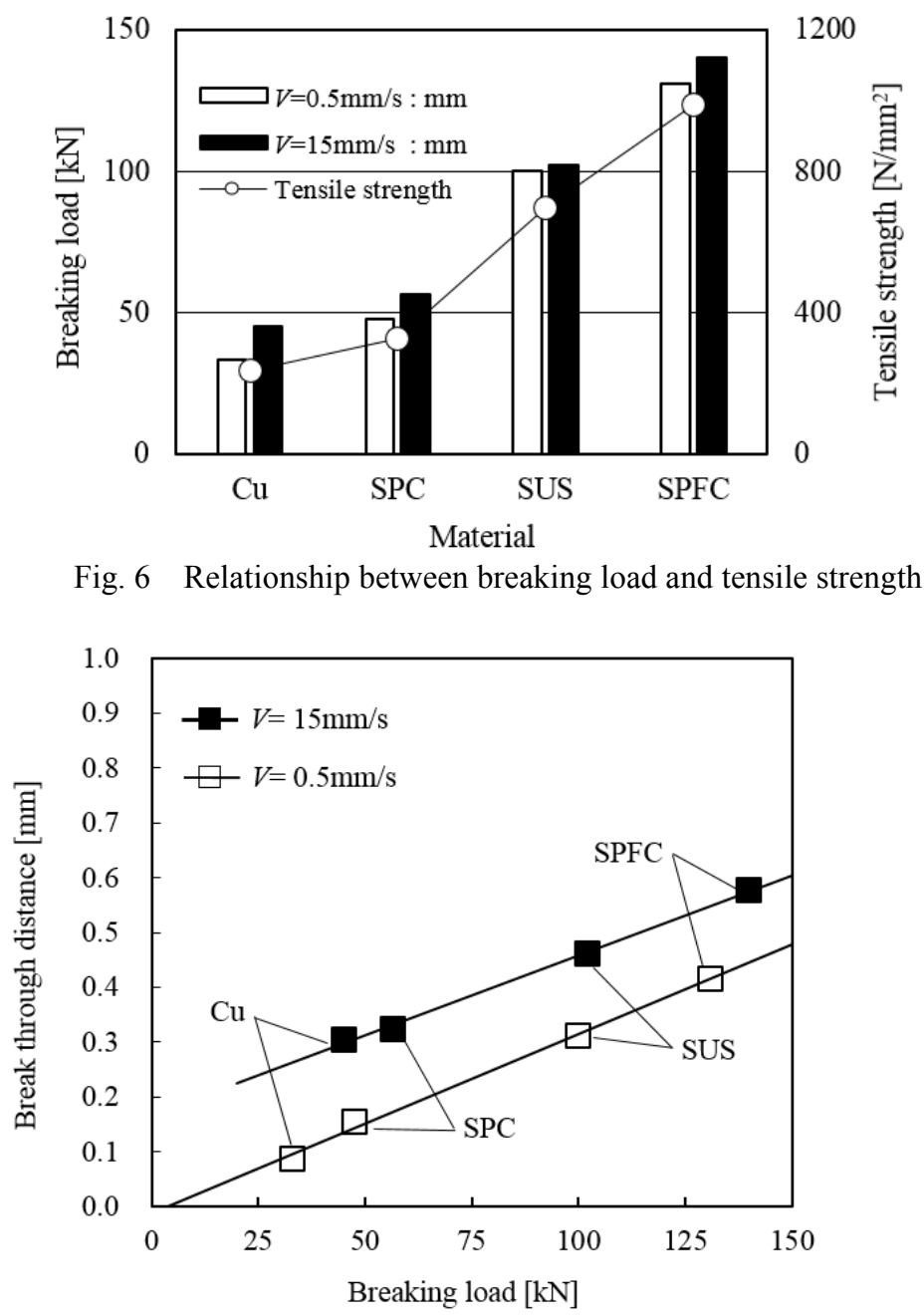

Fig. 7 Relationship between breaking load and break through distance. 


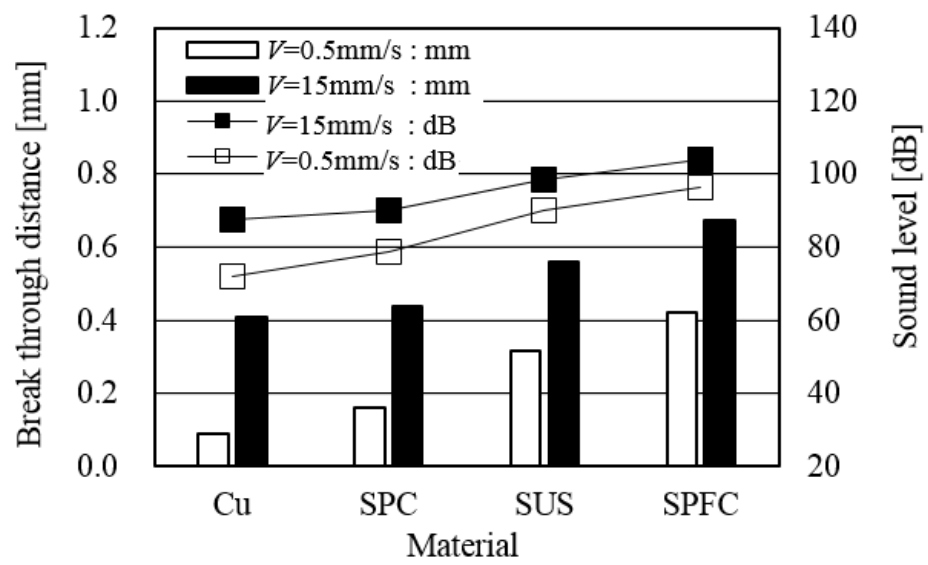

Fig. 8 Relationship between break through distance and sound level

Fig. 6 shows the relationship between the breaking load and the tensile strength of the materials. We defined the point at which the blanking load abruptly deceases as a breaking load. We can observed that the breaking load is proportional to the material strength.

Fig. 7 shows the relationship between the break through distance and the breaking load. The break through distance for the $V=0.5 \mathrm{~mm} / \mathrm{s}$ is proportional to the blanking load. On the other hand, the break through distance for $V=15 \mathrm{~mm} / \mathrm{s}$ is about $0.2 \mathrm{~mm}$ larger than that for $V=0.5 \mathrm{~mm} / \mathrm{s}$. This vibration amount of about $0.2 \mathrm{~mm}$ in $V=15 \mathrm{~mm} / \mathrm{s}$ is almost unrelated in the kind of materials. It means an increase in vibration of a screw drive servo press.

Fig. 8 shows the relationship between the break through distance and the sound level. The sound level is proportional to the break through distance. And it was confirmed that an increase in vibration for $V=15 \mathrm{~mm} / \mathrm{s}$ is related in an increase in sound level for $V=15 \mathrm{~mm} / \mathrm{s}$.

\subsection{Blanking distance}

\subsubsection{Relationship between blanking distance and material}

Fig. 9 shows the blanking distance $l_{b}$ at blanking velocities of $V=15 \mathrm{~mm} / \mathrm{s}$ and $0.5 \mathrm{~mm} / \mathrm{s}$, which is the vibration distance in the $Z$-axial direction from the position at the start of blanking $\mathrm{p} 1$ to the position $\mathrm{p} 2$ of breakage on the blanking trajectory, as shown in Fig.4(a). It was confirmed from Fig. 9 that the $l_{b}$ depends on the material.

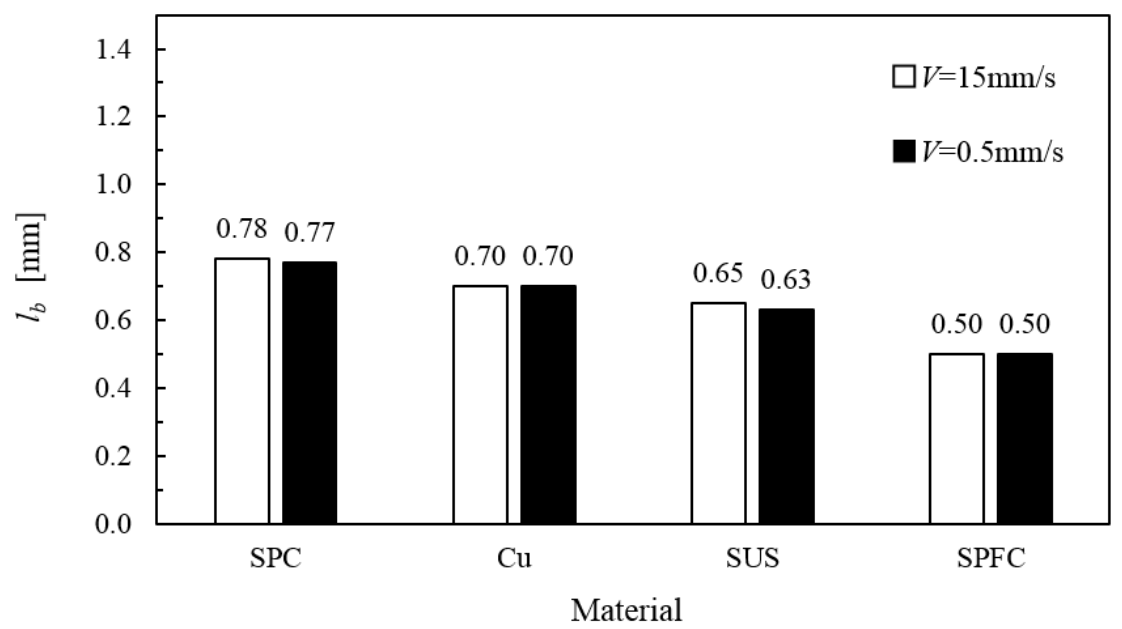

Fig. 9 Blanking distance $l_{b}$ for each material. The blanking distance $l_{b}$ depends on the material.

\subsubsection{Relationship between blanking distance and blanking velocity}

Fig. 10 shows images of the sheared edge of the SPC specimen. Fig. 10 (a) shows a corner of the sheared edge for $V=15 \mathrm{~mm} / \mathrm{s}$, and Fig. 10 (b) shows a linear part obtained at three different velocities to investigate the influence of the blanking velocity, where the image obtained with $V=133 \mathrm{~mm} / \mathrm{s}$ is a product blanked using a crank press. The sheared edge 
in Fig. 10 (b) consists of sheared droop, a sheared surface, and a fracture surface from the upper side. The total distance corresponding to the sheared droop and the sheared surface was about $0.78 \mathrm{~mm}$. It was unrelated of the blanking velocity. This distance $0.78 \mathrm{~mm}$ was almost the same as the blanking distance for SPC indicated in Fig. 9. Also, the blanking velocity was unrelated to the blanking distance in the cases of $\mathrm{Cu}$, SUS, and SPFC. From the above, we confirmed that the blanking velocity is almost unrelated to the blanking distance for $V=15 \mathrm{~mm} / \mathrm{s}$ and $0.5 \mathrm{~mm} / \mathrm{s}$.

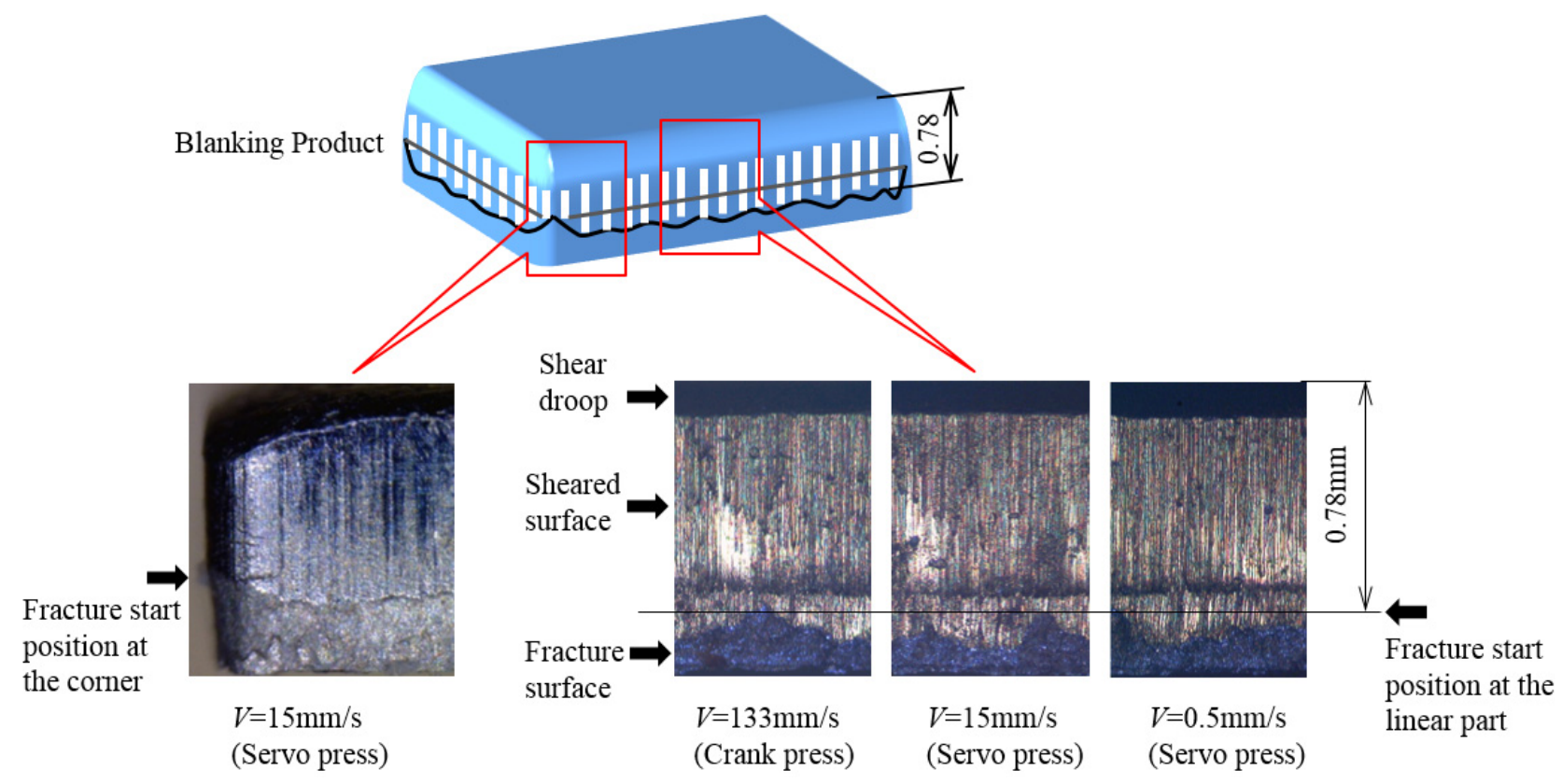

(a) Sheared edge at the corner part

(b) Sheared edge at the linear part

Fig. 10 Sheared edge of SPC (mild steel). The distance of $0.78 \mathrm{~mm}$ indicated in (b) is almost the same as the blanking distance of SPC indicated in Fig. 9. The line of black shadow in the sheared surface in (b) was the same position to the fracture start position at the corner in (a).

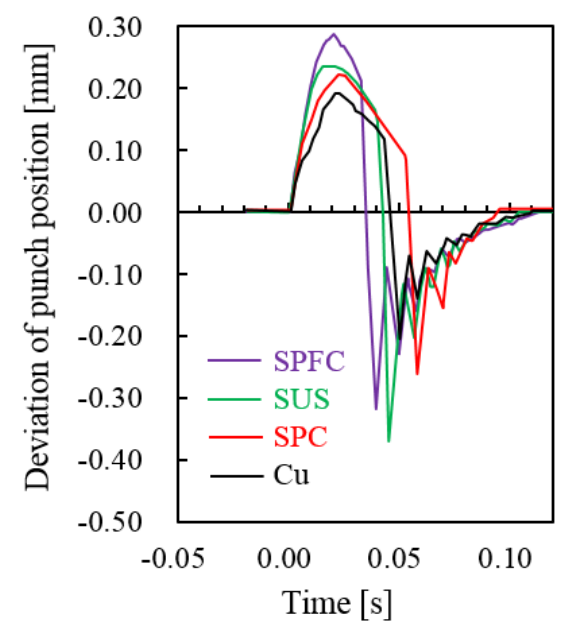

(a) $V=15 \mathrm{~mm} / \mathrm{s}\left(t_{b}<t_{s}\right)$

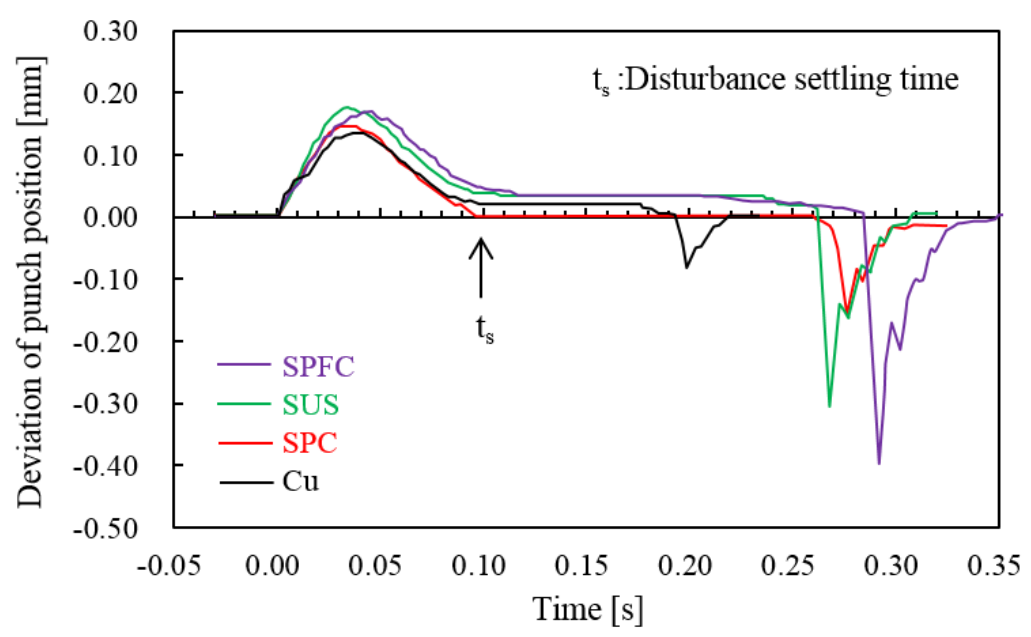

(b) $V=0.5 \mathrm{~mm} / \mathrm{s}\left(t_{b}>t_{s}\right)$

Fig. 11 Deviation of the punch position in blanking, Where deviation = blanking trajectory - planned trajectory. The blanking trajectory and the planned trajectory are shown in Fig. 4. The starting time of the blanking is $0.00 \mathrm{~s}$.

\subsection{Relationship between disturbance settling time and vibration}

When $V=15 \mathrm{~mm} / \mathrm{s}$, the increase in blanking vibration is caused by the response of the feedback control (Murakami, et al., 2015). To confirm the disturbance settling time (Hereafter " $t_{s}$ "), Fig. 11 shows the calculated deviation of the punch 
position. The deviation for $V=0.5 \mathrm{~mm} / \mathrm{s}$ was settled about $0.1 \mathrm{~s}$. On the other hand, in the case of $V=15 \mathrm{~mm} / \mathrm{s}$, the material broke before the deviation was settled. Then we can obtain the $t_{s}$ as about $0.1 \mathrm{~s}$. Therefore, when the blanking time $t_{b}$ is shorter than the $t_{s}$, an increase in blanking vibration occurs as shown in Fig. 11 (a).

We confirmed that a relationship between the disturbance settling time and the blanking vibration. The blanking vibration consists of two factors (Murakami, et al., 2015), the one is the vibration caused by the breaking load, and the other is vibration caused by the control mechanism. Fig. 12 shows details of the factors composing the blanking vibration based on the data in Fig. 7. As shown in Fig. $12(\mathrm{~b})$, when $V=0.5 \mathrm{~mm} / \mathrm{s}, t_{b}$ is longer than $t_{s}$, the vibration caused by the control mechanism is not included. As shown in Fig. 12 (a), when $V=15 \mathrm{~mm} / \mathrm{s}, t_{b}$ is shorter than $t_{s}$, the blanking vibration includes two factors. Thus, the increase in blanking vibration occurred.

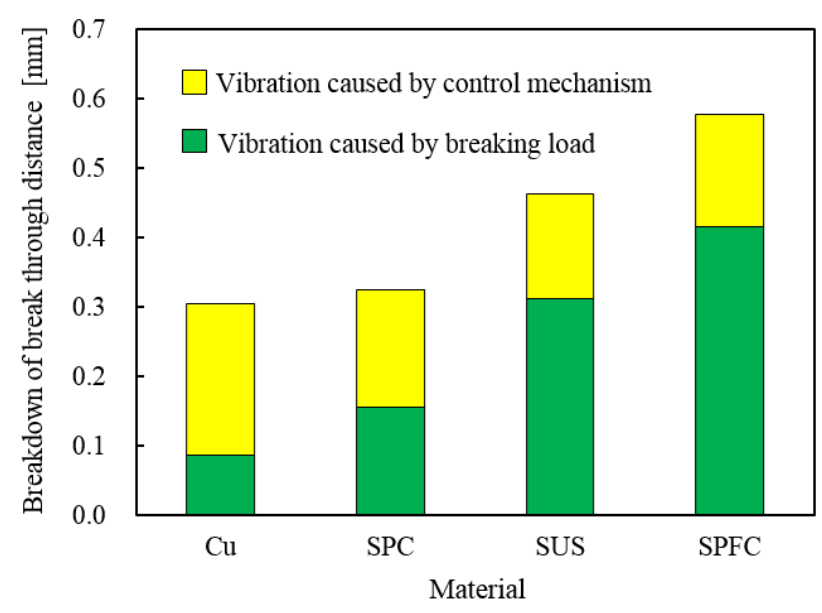

(a) $V=15 \mathrm{~mm} / \mathrm{s}\left(t_{b}<t_{s}\right)$

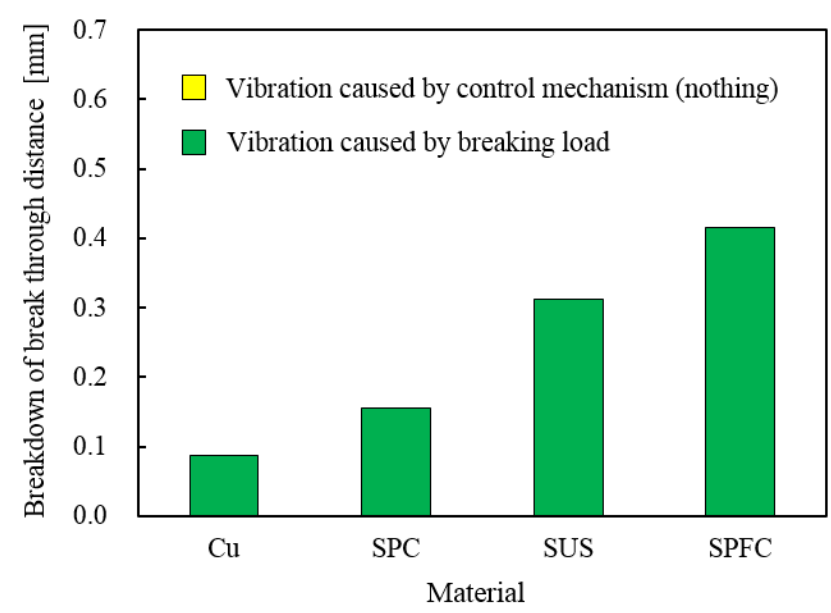

(b) $V=0.5 \mathrm{~mm} / \mathrm{s}\left(t_{b}>t_{s}\right)$

Fig. 12 Breakdown of break through distance using a screw drive servo press. $t_{b}$ : blanking time, $t_{s}$ : disturbance settling time.

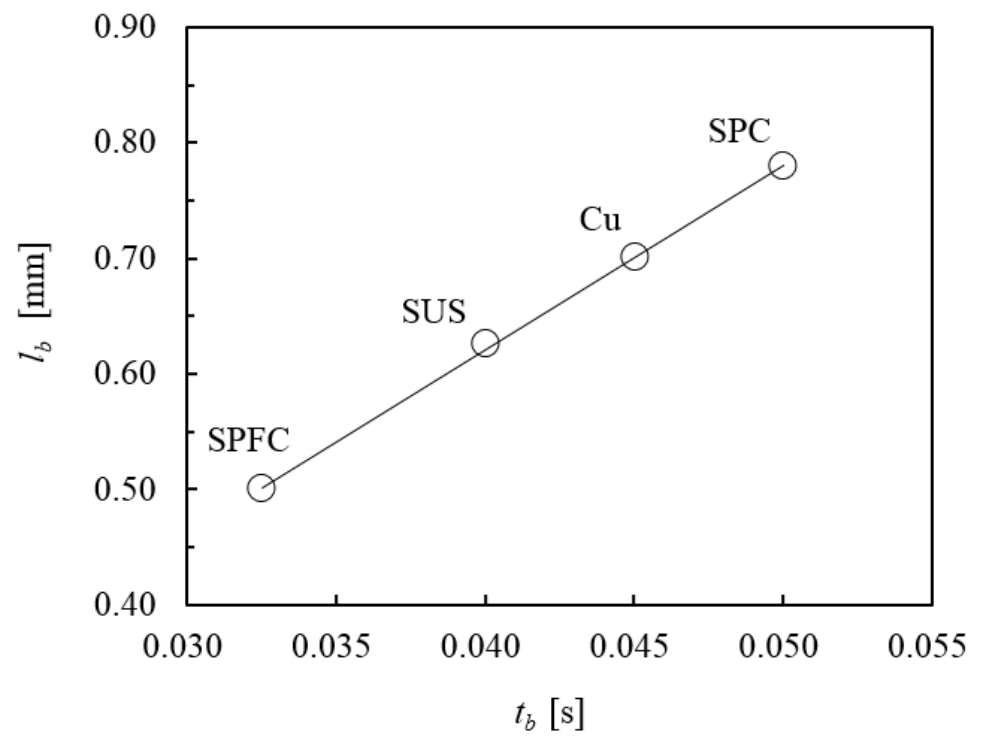

Fig. 13 Relationship between the blanking time $t_{b}$ and the blanking distance $l_{b}$. blanking velocity is $V=15 \mathrm{~mm} / \mathrm{s}$. $l_{b}=15.9 t_{b}-0.01, \quad l_{b}:$ blanking distance $[\mathrm{mm}], \quad t_{b}:$ blanking time $[\mathrm{s}]$.

\subsection{Influence of material on blanking vibration}

Fig. 13 shows the relationship between the blanking distance $l_{b}$ in Fig. 9 and the blanking time $t_{b}$ of each material for $V=15 \mathrm{~mm} / \mathrm{s}$, where the Blanking time is the time from the position $\mathrm{p} 1$ to position $\mathrm{p} 2$ in Fig. 4 . Fig. 13 shows that the $l_{b}$ is 
proportional to the $t_{b}$, where the solid line is the line of best fit calculated by the least-squares method, whose equation is given by to $\mathrm{Eq}$ (1). The intercept of the line of best fit with the vertical axis is -0.01 . Thus, we substitute 0 for the intercept -0.01 . The gradient of 15.9 represents the blanking velocity. As a result, we obtain the Eq (2). The blanking velocity is written as $v$ to generalize Eq. (2), which is transformed into Eq (3) to simulate the blanking time $t_{b}$.

$$
\begin{aligned}
& l_{b}=15.9 t_{b}-0.01 \\
& l_{b}=15.9 t_{b} \\
& t_{b}=\frac{l_{b}}{v}
\end{aligned}
$$

We simulated the blanking time $t_{b}$ for each material using Eq. (3). The calculations condition were set as follows. The blanking velocity $v$ sets it as a constant in the blanking. The $l_{b}$ which was obtained each material data in Fig. 9. The $t_{s}$ was set to $0.10 \mathrm{~s}$. When the $t_{b}$ is shorter than the $t_{s}$, we judge that an increase in blanking vibration occurs.

Fig. 14 shows the result of the simulation. When the $v$ becomes low, the $t_{b}$ becomes long for every material. However, the tendency of the change in the $t_{b}$ depends on the material. $t_{b}>t_{s}$ is only satisfied for SPC when $V=7.7 \mathrm{~mm} / \mathrm{s}$. On the other hand, $t_{b}<t_{s}$ is only SPFC when $V=6.3 \mathrm{~mm} / \mathrm{s}$, because of its low ductility. Therefore, it is necessary to make the blanking velocity lower than for the other materials to prevent an increase blanking vibration for SPFC.

From the above, it was confirmed that the material influences the blanking vibration. To prevent an increase in blanking vibration without losing the productivity when using a screw drive servo press, after obtaining the $l_{b}$ of the material and the $t_{s}$ of the machine, it is necessary to set the threshold of blanking velocity $v_{t}$ so that the $t_{b}$ can be equal to the $t_{s}$, which can be expressed as Eq (4). Further, it is presupposing that a measurement object of $l_{b}$ was punched by the same condition as actual production.

$$
v_{t}=\frac{l_{b}}{t_{s}}
$$

A $v_{t}$ obtained from Eq (4) comes to be the next, $v_{t}$ of SPC is $7.7 \mathrm{~mm} / \mathrm{s}, v_{t}$ of SUS is $6.3 \mathrm{~mm} / \mathrm{s}, v_{t}$ of SPFC is $5 \mathrm{~mm} / \mathrm{s}$. These values of $v_{t}$ are the point of intersection with the line of $t_{s}=0.10 \mathrm{~s}$ and the line of each material on Fig. 14 . We checked with Fig. 15 to inspect these values of $v_{t}$. Fig. 15 shows relationship between blanking velocity and increase in blanking vibration. A threshold of blanking velocity can be confirmed clearly. These values in Fig.15 are almost same as values of $v_{t}$ that we obtained from Eq (4). Therefore, We propose Eq (4) as a general formula which calculates $v_{t}$.

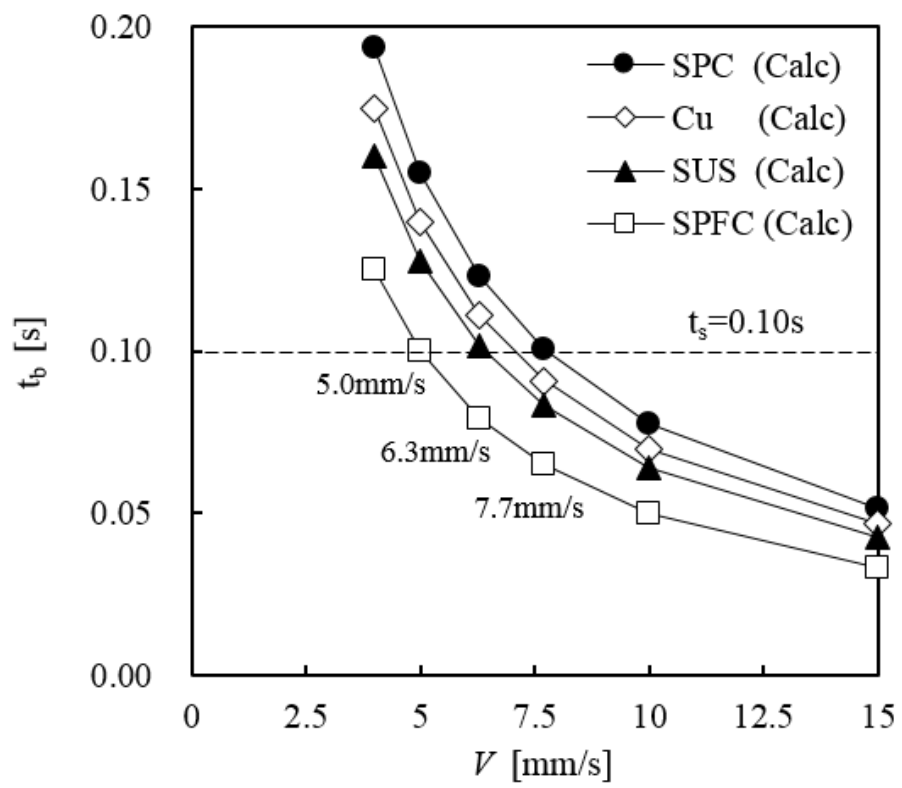

Fig. 14 Relationship between $t_{b}$ and materials obtained by simulation using Eq. (3). $t_{b}$ : blanking time, $t_{s}$ : disturbance settling time, $V$ : blanking velocity. The dashed line represents $t_{s}=0.10 \mathrm{~s}$. When $t_{b}$ is shorter than $t_{s}$, an increase in blanking vibration occurs. 


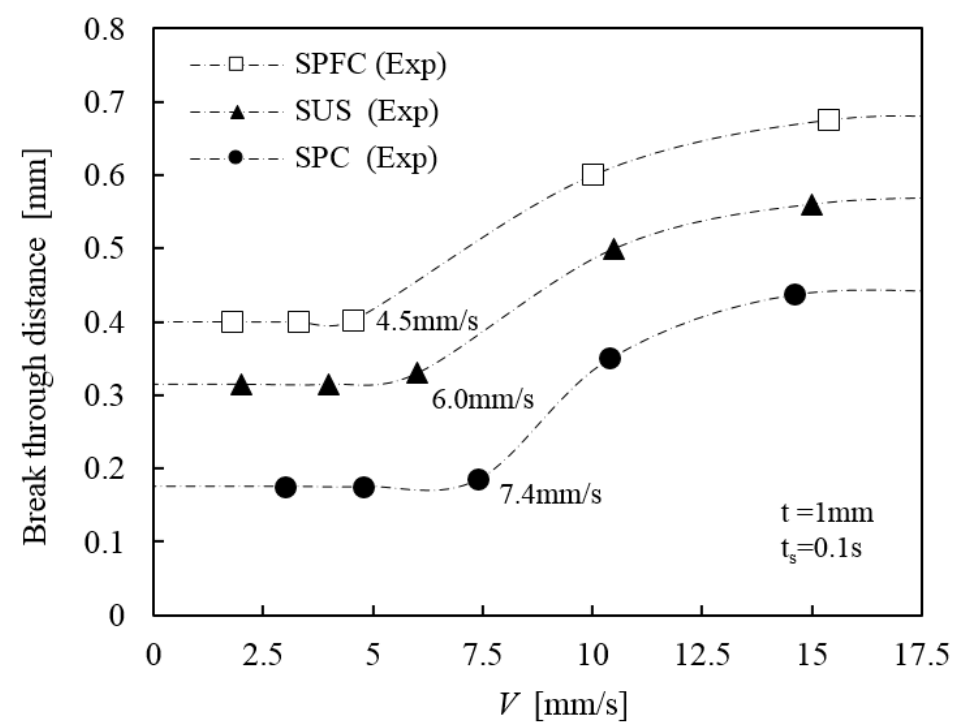

Fig. 15 Relationship between blanking velocity and increase in blanking vibration. The blanking condition is same as Fig.4. $V$ : blanking velocity. A data of $V$ was obtained from average velocity of $\mathrm{p} 1$ to $\mathrm{p} 2$ in a blanking trajectory such as Fig.4, Fig.5. It was confirmed a threshold of blanking velocity clearly in this figure.

\section{Conclusion}

The following points summaries the influence of the material on the blanking vibration using a screw drive servo press.

(1) When the blanking time $t_{b}$ is longer than the disturbance settling time $t_{s}$, an increase in blanking vibration does not occur. Under this condition, the blanking vibration is caused by the breaking load of the material.

(2) When the blanking time $t_{b}$ is shorter than the disturbance settling time $t_{s}$, an increase in blanking vibration occurs. Under this condition, the blanking vibration consists of two factors: vibration caused by the breaking load, and vibration caused by the control mechanism.

(3) The blanking distance $l_{b}$ depends on the material. In the case of the same material, the blanking velocity is almost unrelated to the blanking distance when $V=0.5 \mathrm{~mm} / \mathrm{s}$ to $15 \mathrm{~mm} / \mathrm{s}$.

(4) From the above, We propose Eq (4) as a general formula which calculates the threshold of blanking velocity $v_{t}$ to prevent an increase in blanking vibration.

\section{References}

Ghiotti, A., Regazzo, P., Bruschi, S., Bariani , P.F., Reduction of vibrations in blanking by MR dampers, CIRP Annals - Manufacturing Technology, Vol.59, Issue 1, (2010), pp.275-278.

JIS Z2241:2011, Japanese Industrial Standards, Metallic materials-Tensile testing-Method of test at room temperature,(2011), (in Japanese).

Machida, T., Murata, C., Hasegawa, K., Development of digital Servo press, Journal of the Japan Society of Electrical Machining Engineers, Vol.39, No.90 (2005), pp.43-47 (in Japanese).

Murakami, T., Mechanism behind increase in vibration in blanking using screw drive servo press, Journal of JSTP, vol.56, No.651 (2015), pp. 305-310 (in Japanese).

Murakawa, M., Mo, J., Wakatsuki, Y., Koga, N., Investigation of blanking noise reduction using a hydraulic inertia damper, Journal of Materials Processing Technology, Vol.112, Issue 2-3, (2001), pp.205-213.

Osakada, K., Mori, K., T, Altan., P, Groche., Mechanical servo press technology for metal forming, CIRP Annals Manufacturing Technology, Vol.60, issue2 (2011), pp.651-672.

Otsu, M., Yamagata, C., Osakada, K., Reduction of Blanking Noise by Controlling Press Motion, CIRP Annals Manufacturing Technology, Vol.52, issue1 (2003), pp.245-248.

Suzuki, A., Ac servo press HCP3000, Journal of Press Technology, Vol.39, No.10 (2001), pp.37-39 (in Japanese). 